\section{Comment on 'Statement on combined hormonal contraceptives containing third- or fourth-generation progestogens or cyproterone acetate, and the associated risk of thromboembolism'}

Despite the increased interest in evidence-based medicine, many medical guidelines and statements of medical professionals are based primarily on opinion rather than scientific facts. The 'Statement on combined hormonal contraceptives containing third- or fourthgeneration progestogens or cyproterone acetate, and the associated risk of thromboembolism' raises such concerns. ${ }^{1}$

While we agree that the importance of effective and well-tolerated contraceptives is indisputable, we must ask for evidence that questioning the safety of newer-generation progestogens compared to older formulations has caused a "new pill scare", or a "crisis" resulting in a "highly emotional political dimension". "The implication that ideology or overreaction rather than scientific analysis underlies the numerous recent calls for increased scrutiny and changes in prescribing practice, and that patients are being harmed as a result, deserves a serious response.

Contraceptives improve women's lives and their health, and there is no question that all contraceptive methods have lower thromboembolic risks than pregnancy. However, given that all combined oral contraceptives have similar efficacy in terms of pregnancy prevention, the public health question should be what differences in adverse reactions are acceptable, to patients, doctors and regulatory government entities. Clearly the nature of the adverse effect is the crucial issue; patients and their physicians may be willing to tolerate numerous minor side effects such as spotting and breast tenderness, but a statistically significant increased risk of potentially fatal blood clots in most independent studies deserves the attention of all physicians. For example, the largest study to date found an increase in the hazard ratio (HR) for venous thromboembolism among new users of drospirenone contraceptives in relation to other low-dose estrogen comparators (HR 1.77; 95\% confidence interval $1.33-2.35){ }^{2}$

Even a small, significant additional risk is greatly magnified because millions of women and girls use contraceptives. Are any additional deaths and disabilities acceptable, to patients or their doctors, when so many safer, equally effective oral contraceptives are available? The authors posit that women "need a large spectrum of options ... in order to tailor contraceptive choice to individual women's needs", but how large does that spectrum need to be? Scores of different brand name and generic oral contraceptives are sold in the USA, and the French Health Plan pays for at least a dozen different products that do not contain drospirenone. ${ }^{3}$ Our review of all the published studies has failed to find any evidence that it is necessary to offer more than a dozen of the safer types of oral contraceptives to maximise patient satisfaction, contraceptive use or compliance.

Physician behaviour has been shown to be influenced by financial relationships with pharmaceutical companies, from the level of individual prescribing practice to consensus guidelines. ${ }^{4}$ In the menopausal hormone therapy literature, for example, a connection has been observed between receiving industry funding for speaking, consulting or research and the publication of promotional opinion pieces. ${ }^{5}$ Given that almost all of the 25 Statement authors reported conflicts of interest, their strongly expressed hostility toward physicians who convey concerns about contraceptives with significantly riskier profiles in numerous well-designed studies seems inappropriate for a medical journal.

\section{Mishka Terplan, MD, MPH}

National Research Center for Women \& Families, Washington, DC, USA; mt@center4research.org

\section{Diana Zuckerman, PhD}

National Research Center for Women \& Families, Washington, DC, USA; dz@center4research.org

Competing interests None.

Provenance and peer review Not commissioned; internally peer reviewed.

J Fam Plann Reprod Health Care 2013;39:304. doi:10.1136/jprhc-2013-100693

\section{REFERENCES}

1 Blitzer J, Amy JJ, Beerhuizen R, et al. Statement on combined hormonal contraceptives containing third- or fourthgeneration progestogens or cyproterone acetate, and the associated risk of thromboembolism. J Fam Plann Reprod Health Care 2013;39:156-159.

2 Sidney S, Cheetham TC, Connell FA, et al. Recent combined hormonal contraceptives (CHCs) and the risk of thromboembolism and other cardiovascular events in new users. Contraception 2013;1:93-100.

3 Watkins ES. How the pill became a lifestyle drug: the pharmaceutical industry and birth control in the United States since 1960. Am J Public Health 2012;8:1462-1472.

4 Newman J, Korenstein D, Ross JS, et al. Prevalence of financial conflicts of interest among panel members producing clinical practice guidelines in Canada and United States: cross sectional study. BMJ 2011;343: d5621.

5 Fugh-Berman A, McDonald CP, Bell AM, et al. Promotional tone in reviews of menopausal hormone therapy after the Women's Health Initiative: an analysis of published articles. PLoS Med 2011;3: e1000425. 